\title{
A tool for neutrophil guided dose adaptation in chemotherapy
}

\author{
Johan E. Wallin*, Lena E. Friberg, Mats O. Karlsson \\ Department of Pharmaceutical Biosciences, Uppsala University, BMC Box 591, 75184 Uppsala, Sweden
}

\section{A R T I C L E I N F O}

Article history:

Received 3 July 2008

Received in revised form

15 October 2008

Accepted 24 October 2008

\section{Keywords:}

Myelosuppression

Dose adaptation

Adaptive control

Bayesian forecasting

Pharmacodynamics

Neutropenia

\begin{abstract}
A B S T R A C T
Chemotherapy dosing in anticancer treatment is a balancing act between achieving concentrations that are effective towards the malignancy and that result in acceptable side-effects. Neutropenia is one major side-effect of many antitumor agents, and is related to an increased risk of infection. A model capable of describing the time-course of myelosuppression from administered drug could be used in individual dose selection. In this paper we describe the transfer of a previously developed semi-mechanistic model for myelosuppression from NONMEM to a dosing tool in MS Excel, with etoposide as an example. The tool proved capable to solve a differential equation system describing the pharmacokinetics and pharmacodynamics, with estimation performance comparable to NONMEM. In the dosing tool the user provides neutrophil measures from a previous treatment course and request for the dose that results in a desired nadir in the upcoming course through a Bayesian estimation procedure.
\end{abstract}

() 2008 Elsevier Ireland Ltd. All rights reserved.

\section{Introduction}

Neutropenia is a major side-effect of many antitumor agents, and is often the dose-limiting factor [1] The risk of infection is related to the degree and duration of neutropenia $[2,3]$. Dose adjustment to minimize the risk of experiencing severe neutropenia could either be made a priori or a posteriori, ranging in complexity from body size based dosing up to utilization of statistical models and various sampling. In this paper we show implementation of a pharmacokinetic-pharmacodynamic model, describing the neutrophil concentrations after anti-cancer treatment, in a simple tool to be used in the patient care at the clinic. This tool would facilitate use of neutrophil data from the first completed course of treatment in a Bayesian maximum a posteriori process to guide dose selection in an upcoming course. Etoposide was chosen as a model substance, due to it being rather well-characterized and its wide-spread use, and the access to a suitable validation dataset.

A predictive model of myelosuppression could be used for dose individualization, in order to avoid neutropenia, a prolonged treatment interval and/or a suboptimal tumor effect. For etoposide current treatment guidelines state that occurrence of severe neutropenia should lead to a reduction or postponement of next dose until neutrophil recovery [4]. The dose reduction is not necessarily tailored to the patient, but usually by fixed decrements such as to $75 \%$ or $50 \%$ of the original dose. This leads to a one-sided dose adjustment, where only patients experiencing toxicity receives a dose change, but not necessarily in an optimal fashion. The possibility that patients experiencing only a minor myelosuppression are underexposed and might benefit from an increased dose is not taken into account. The relation between hematological toxicity and patient survival has been investigated in clinical

\footnotetext{
* Corresponding author at: Division of Pharmacokinetics and Drug Therapy, Biomedical Centre, Uppsala University, Sweden. Tel.: +4618 471 4639; fax: +4618471 4003 .

E-mail address: johan.wallin@farmbio.uu.se (J.E. Wallin). 0169-2607/\$ - see front matter @ 2008 Elsevier Ireland Ltd. All rights reserved. doi:10.1016/j.cmpb.2008.10.011
} 
studies of breast cancer and non-small cell lung cancer, suggesting that a grades $2-3$ neutropenia provides an increased patient survival compared to milder or more severe toxicity [5-7].

Numerous attempts to define a priori dosage adjustments of etoposide has been made and predominantly such dosing algorithms base dosage on renal function $[8,9]$ or hepatic function [10-12]. A more refined way to adjust dosing has been the application of a maximum a posteriori (MAP) Bayesian guided dosing, utilizing knowledge of the population distribution of the pharmacokinetic parameters for the drug [13-15]. By using drug concentration measurements from a patient and a population pharmacokinetic model, the most likely parameters for the individual at hand can be obtained by optimization of a Bayesian function. These parameters can then be used to calculate the dose intended to result in a target drug exposure best correlated to the desired effect in the population. MAP Bayesian pharmacokinetic guided dosing has been developed for numerous anticancer drugs, for a wider overview of the area, see Rousseau et al. [16]. We here propose a method for utilizing pharmacodynamic rather than pharmacokinetic data.

While pharmacokinetics can be described as the characteristics of the dose-concentration response, pharmacodynamics relates concentrations to effects. Inter-individual variability in the pharmacodynamics is generally thought to be larger than the inter-individual variability in pharmacokinetics [17]. This has also been shown in chemotherapy-induced myelosuppression $[18,19]$. With a model involving both observed plasma drug concentration and neutrophil counts from the first cycle, not only the pharmacokinetic parameters of the patient can be assessed, but also the pharmacodynamic parameters of the dose-limiting toxicity, and thus drug therapy can be optimized on an even more individualized level. A predictive pharmacokinetic-pharmacodynamic (PKPD) model of myelosuppression may use the individual pharmacokinetic parameters, the individual pharmacodynamic system parameters as well as an estimate of the individual's drug sensitivity.

Several attempts have been made to characterize the relations between dose of anticancer drugs and concentration-time profile of neutrophils [20]. A previously published semi-physiological model for myelosuppression [21] has successfully been applied for several cytotoxic drugs, one of them being etoposide. The model is composed of three transit compartments linking the maturation and proliferation from the stem cell pool in bone marrow to circulating neutrophils and a feedback function mimicking the G-CSF mediated bone-marrow regulation. Drug effect can often be sufficiently described by a linear function relating the plasma concentration to inhibition of cell proliferation and cell kill in the proliferation pool. The model is semi-mechanistic in the sense that it does not fully represent all the maturation and proliferation steps, but still captures the main physiological processes. It is parameterized in terms such as mean transit time (MTT) and neutrophil baseline levels (BASE). The third system parameter (GAMMA) characterizes the magnitude of the feedback function in which the current level of neutrophils is related to the estimated baseline neutrophil count (Fig. 1). The drug-specific toxicity parameter is denoted SLOPE. This sort of transit compartment model is nonlinear and has no simple analytical solution, but can be easily described with differential equations. Modeling of differential equation systems can be done in software containing a differential equation solver.

A pharmacokinetic-pharmacodynamic model with good predictability from one course to the next might benefit dose selection in patients, hence implementing the model in a clinical setting as a tool for dose adaptation. The semi-mechanistic model has been shown to be of good aid in clinical drug development and study design [22], see for example [19,23-27], but has not been applied in day-to-day patient care. A study across several anti-cancer agents also reveals that interindividual variability (IIV) is overall significantly larger than inter-occasion variability (IOV) [28], which facilitates Bayesian forecasting. Results for etoposide suggest that it is significant to include both IIV and IOV in the parameters MTT and SLOPE, but for BASE only IIV was significant to include. For MTT, coefficient of variation for IIV was $23 \%$ compared to an IOV of $12 \%$. For SLOPE IIV was $28 \%$ and IOV was $39 \%$. IIV in BASE was $47 \%$. Simulations from these results showed total IIV to be of significantly smaller magnitude than IOV.

\section{Computational methods and theory}

The most commonly used software for non-linear mixedeffect modeling in pharmacokinetic and pharmacodynamic modeling is NONMEM (Globomax) [29]. The myelosuppres-

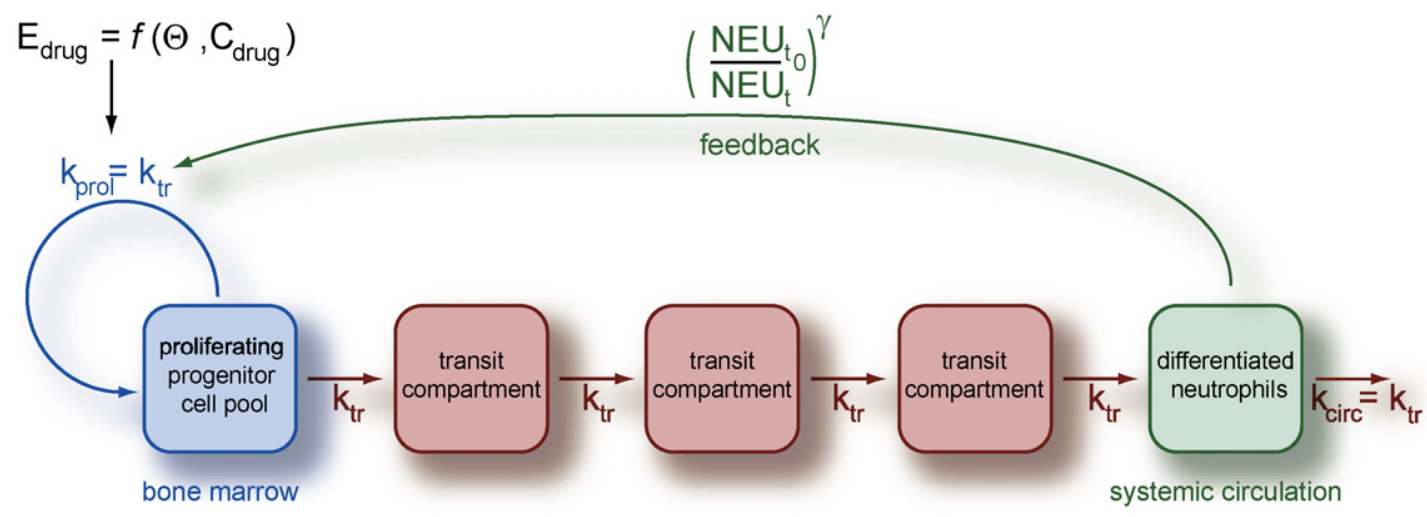

Fig. 1 - Description of the semi-physiological model of myelosuppression. 
sion model has been developed in this environment, and dose optimization could very well be carried out using this software, but there are several incentives for moving to another environment; NONMEM and any other specialized software that can handle non-linear mixed effect modeling have a high knowledge threshold for use and highly specific demands for data input. These matters would become important if aiming towards distributing the dosing tool to clinics, where it will be used by physicians, nurses or pharmacists. A shell to the software could overcome the data input and operation difficulties but this would consider licensing, and a cost free distribution would not be likely.

Our solution was to implement this method into MS Excel (MS Excel 2003, Microsoft Corp., Seattle, USA), it is installed on a majority of all workstations, contributes a familiar environment, and can manage the desired mathematics and graphics. The PKPD-model unfortunately cannot be solved analytically, something that would significantly have reduced computation time, and made the use of add-ins unnecessary. MS Excel standard installation cannot solve differential equations, but an add-in called PopTools (CSIRO) [30] contains a differential equation solver capable of handling large equation systems. As NONMEM is the gold standard for this type of implementation, one demand for the Excel workbook would be to perform similarly to NONMEM, in terms of ability to predict future measurements and individual parameters.

\subsection{Pharmacokinetic-pharmacodynamic model}

We have exemplified the implementation of a posteriori dose adaptation based on neutrophil counts with the cytotoxic drug etoposide. The tool is however able to technically implement any drug characterized with the myelosuppression model. To describe the pharmacokinetics of etoposide, a twocompartment pharmacokinetic model published by Toffoli et al was applied [31] and the pharmacodynamics was based a the publication of the semi-physiological model of myelosuppression by Friberg et al. [21]. Interindividual variability was included on BASE, MTT and SLOPE without correlations (Table 1).

\subsection{The tool}

The model consists of a differential equation for every pharmacokinetic compartment (two PK compartments were needed for etoposide), and five equations describing the neutrophil dynamics.

Pharmacokinetic system:

$$
\begin{aligned}
\frac{\mathrm{dA}(1)}{\mathrm{dt}}= & \sum_{0}^{n}\left\{k_{\text {inf }_{d}} \times\left[1-\operatorname{HEAV}\left(\mathrm{t}_{d}-\mathrm{t}_{0_{d}}-\mathrm{t}_{\text {inf }_{d}}\right)\right]\right\} \\
& -\left(\frac{\mathrm{CL}}{\mathrm{V} 1} \times \mathrm{A}(1)\right)-\left(\frac{\mathrm{Q}}{\mathrm{V} 1} \times \mathrm{A}(1)\right)+\left(\frac{\mathrm{Q}}{\mathrm{Vss}} \times \mathrm{A}(2)\right)
\end{aligned}
$$

$$
\frac{\mathrm{dA}(2)}{\mathrm{dt}}=\left(k_{12} \times \mathrm{A}(1)\right)-\left(k_{21} \times \mathrm{A}(2)\right)
$$

where $n$ is the number of doses in a patient, $k_{\text {inf }_{d}}, t_{0_{d}}$ and $t_{d}$ are the infusion rate, the start time and the duration of the
Table 1 - Model parameter estimates used for simulation and estimation.

\begin{tabular}{llc} 
Parameter & \multicolumn{1}{c}{ Typical value } & $\begin{array}{c}\text { Interindividual } \\
\text { variability }\end{array}$ \\
\hline CL $(\mathrm{L} / \mathrm{h})$ & 1.14 & $25 \%$ \\
$\mathrm{~V}_{1}(\mathrm{~L})$ & 6 & $25 \%$ \\
$\mathrm{k}_{12}\left(\mathrm{~h}^{-1}\right)$ & 0.14 & $21 \%$ \\
$\mathrm{~K}_{21}\left(\mathrm{~h}^{-1}\right)$ & 0.06 & $29 \%$ \\
$\mathrm{~F}$ & 0.44 & $22 \%$ \\
Residual error PK & 0.214 additive & \\
& $18.7 \%$ proportional & \\
& & \\
BASE & 5.45 & $42 \%$ \\
MTT & 135 & $14 \%$ \\
SLOPE & 0.126 & $40 \%$ \\
GAMMA & 0.174 & \\
Residual error PD & 0.671 additive \\
& $45.3 \%$ proportional \\
\hline
\end{tabular}

CL is the amount of blood totally cleared from drug per time unit, $V_{1}$ is the apparent volume of distribution of the central compartment, $k_{12}$ and $k_{21}$ is the transition rates of drug to and from, respectively, the peripheral compartment and $\mathrm{F}$ denotes the bioavailability.

$\mathrm{d}^{\text {th }}$ administration, CL is the plasma clearance of drug, $Q$ is the intercompartmental clearance between the central compartment and the peripheral compartment, $\mathrm{V} 1$ is the apparent volume of distribution of the central compartment, Vss is the apparent volume of distribution at steady state, and $A(1)$ and $A(2)$ are the drug amounts in respective compartments. HEAV is a Heaviside function, i.e. a function that alters between 0 and 1 at given time points. The function was here used to introduce drug into the central compartment during the time of infusion.

Pharmacodynamic system:

$$
\begin{aligned}
\frac{\mathrm{dA}(3)}{\mathrm{d} t}= & {\left[k_{\operatorname{tr}} \times \mathrm{A}(3) \times\left\{1-\left(\frac{\mathrm{SLOPE} \times \mathrm{A}(1)}{\mathrm{V} 1}\right)\right\}\right.} \\
& \left.\times\left(\frac{\mathrm{BASE}}{\mathrm{A}(7)}\right)^{\text {GAMMA }}\right]-k_{\operatorname{tr}} \times \mathrm{A}(3)
\end{aligned}
$$

$$
\begin{aligned}
& \frac{\mathrm{dA}(4)}{\mathrm{dt}}=k_{\mathrm{tr}} \times \mathrm{A}(3)-k_{\mathrm{tr}} \times \mathrm{A}(4) \\
& \frac{\mathrm{dA}(5)}{\mathrm{dt}}=k_{\mathrm{tr}} \times \mathrm{A}(4)-k_{\mathrm{tr}} \times \mathrm{A}(5) \\
& \frac{\mathrm{dA}(6)}{\mathrm{dt}}=k_{\mathrm{tr}} \times \mathrm{A}(5)-k_{\mathrm{tr}} \times \mathrm{A}(6) \\
& \frac{\mathrm{dA}(7)}{\mathrm{dt}}=k_{\mathrm{tr}} \times \mathrm{A}(6)-k_{\text {circ }} \times \mathrm{A}(7)
\end{aligned}
$$

where $A(3)$ consists of immature cells in the proliferation pool; $A(4), A(5)$ and $A(6)$ represent the non-mitotic maturing cells in the transit compartments and $A(7)$ is the concentration of circulating neutrophils. $k_{t r}$ is the transit rate constant, derived from 4/MTT as there are four transits from the proliferative to the circulating compartment, and $k_{\text {circ }}$ derives from the $t 1 / 2$ of circulating neutrophils $(7 \mathrm{~h})[32], k_{\text {circ }}=\ln 2 / t_{1 / 2}$. 


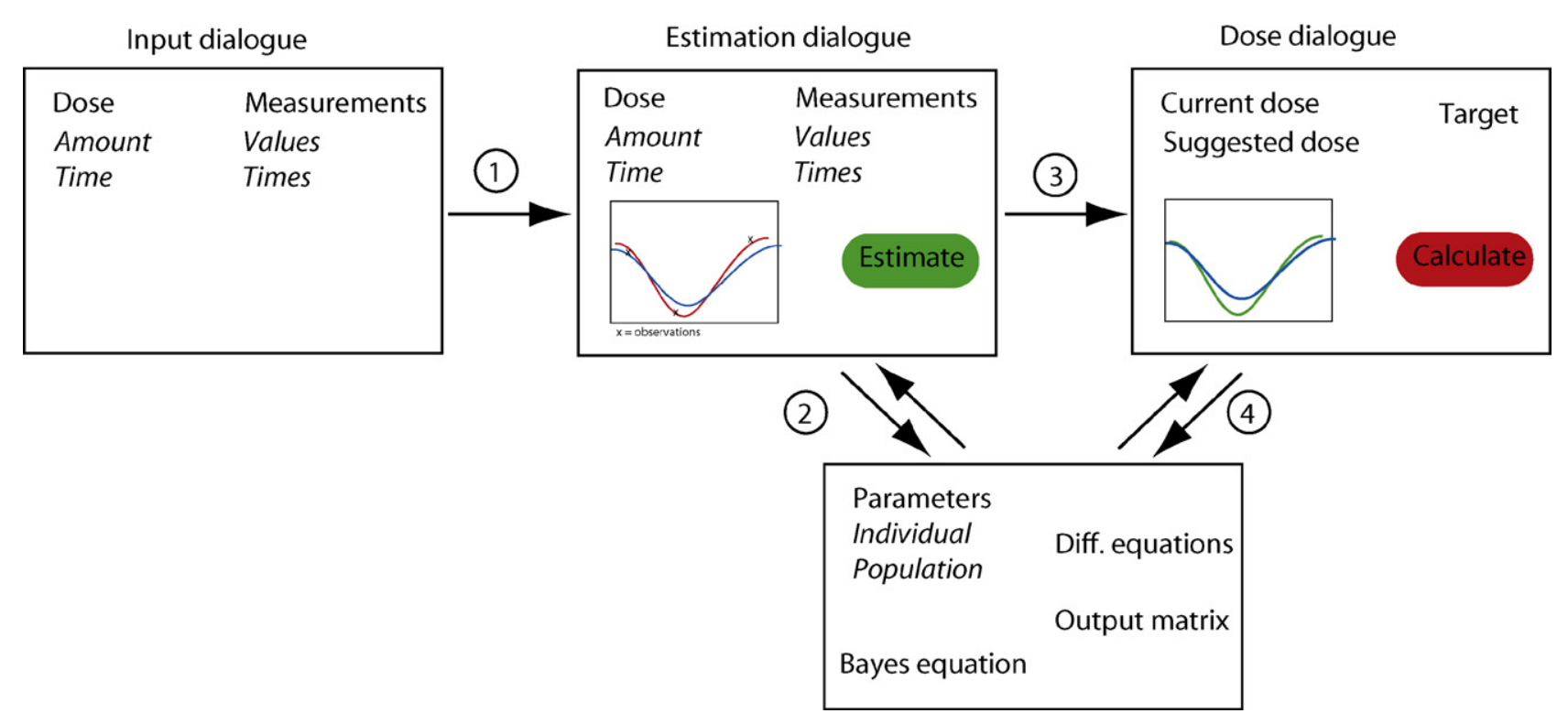

Fig. 2 - The structure of the workbook and the process order; (1) Graphical presentation of indata, (2) the Baeysian estimation, (3) input of desired treatment target and (4) the calculation of an individualized dose to obtain the desired target.

A MAP Bayesian process iterates in order to minimize the objective function [33-35]:

$\mathrm{OBJ}_{\mathrm{MAP}}=\sum_{j=1}^{p}\left[\frac{\hat{P}_{j}-P_{j}}{\omega_{j}}\right]^{2}+\sum_{i=1}^{m}\left[\frac{\hat{C}_{i}-C_{i}}{\sigma_{i}}\right]^{2}$

where $p$ is the number of parameters, $P_{j}$ is the typical value of the $j^{\text {th }}$ parameter in the population, $\omega_{j}$ represent the interindi- vidual variability of the parameter, $\hat{P}_{j}$ is the estimate of the individual's parameter, $\mathrm{m}$ is the number of observations, $C_{i}$ is the observed plasma drug concentration or neutrophil count, $\hat{\mathrm{C}}_{i}$ is the predicted measurements using the $\hat{\mathrm{P}}_{j}$ values, and $\sigma_{i}$ is the residual variability of the observations.

The model was successfully implemented in a MS Excel workbook of approximately one megabyte in size, consisting of four worksheets. POPTOOLS proved able to solve the dif-

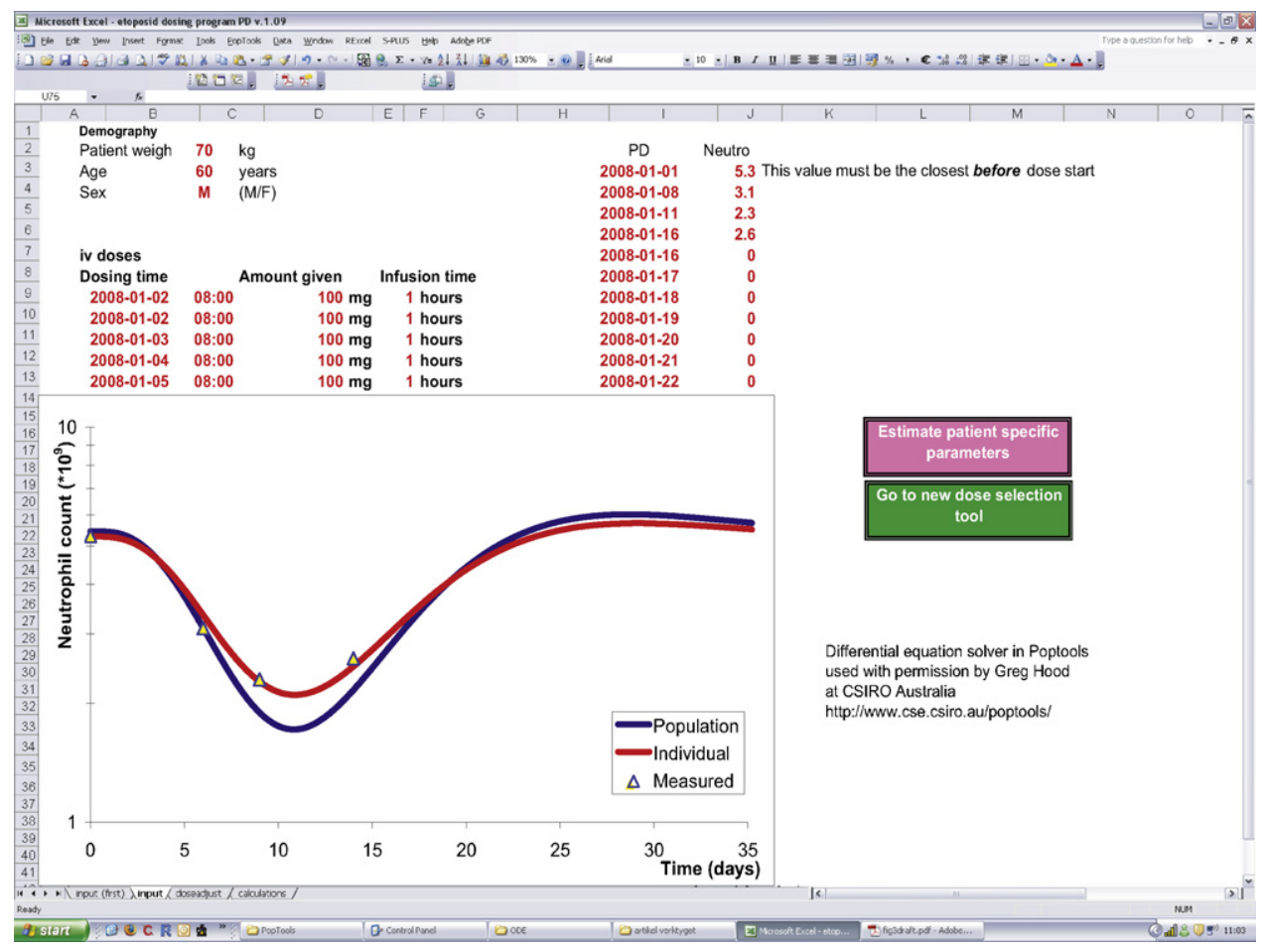

Fig. 3 - Dose and measurement input dialogue with graphical visualization of time-course of neutrophils for the specific vs a typical individual receiving the administered dose. 


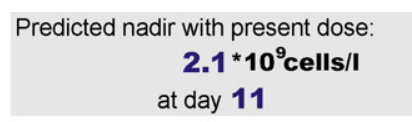

$\begin{array}{cc}\text { Suggested new dose: } & \text { Inf. time: } \\ 182 \mathrm{mg} & 1 \text { hours } \\ 182 \mathrm{mg} & 1 \text { hours } \\ 182 \mathrm{mg} & 1 \text { hours } \\ 182 \mathrm{mg} & 1 \text { hours } \\ 182 \mathrm{mg} & 1 \text { hours }\end{array}$

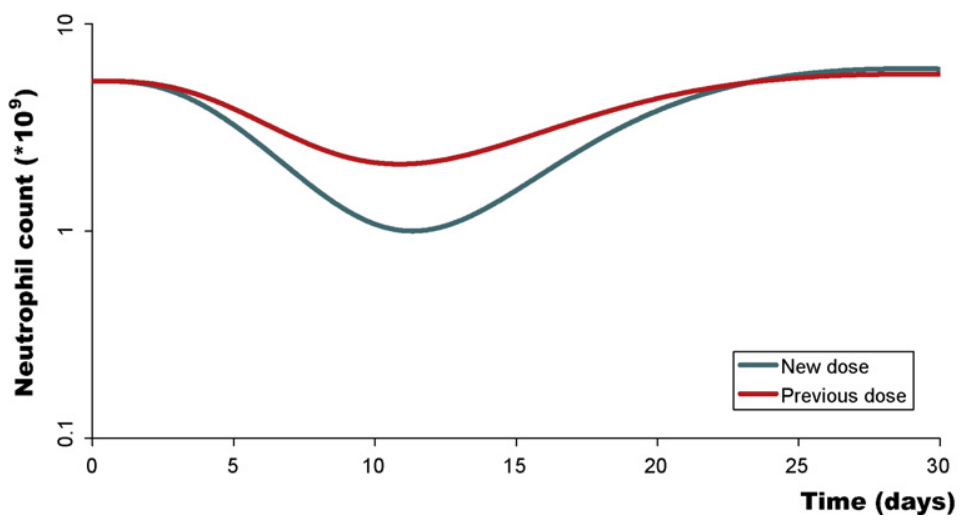

Suggest new dose

Reset dose

Will result in nadir of $1.0 * 10^{9} \mathrm{cells} / \mathrm{l}$

At day 11

Go back to data input

Fig. 4 - Dose suggestion worksheet with graphical visualization of predicted time-course of neutrophils for the current individual receiving the previous and the suggested dose.

ferential equation system, and the use of Heaviside functions facilitated the use of multiple doses. One version of the tool was also constructed to handle oral administration, by the addition of absorption compartments.

\section{Program description}

The flow of information of the tool is depicted in Fig. 2. The workbook was constructed to show an input dialogue where dose time and dose size as well as sample times, concentration measurements and/or neutrophil measurements are entered. This information is automatically transferred to another worksheet where also the predicted neutrophil-time profile is shown. By the click of a button a Bayesian estimation process is started which minimizes an objective function value (OBJ $\mathrm{MAP}_{\mathrm{P}}$ ) (Eq. (8)), by changing the individual system and drug specific parameters of the model. More specifically the differential equation solver outputs a matrix with the amounts in every compartment over one thousand time points. A lookup function is used to match every measurement input by the user, with its prediction in the output matrix. The sum of these residuals is part of the $\mathrm{OBJ}_{\mathrm{MAP}}$. The macro utilizes the built-in Solver function, to minimize the OBJ $J_{M A P}$ by searching for plausible parameters by an iterative procedure. In every iteration, the residuals in the output matrix are updated. This Bayesian procedure can be tuned in regards to what parameters to be estimated can be defined, as well as boundary values. The graphics in the estimation dialogue now display both the predicted neutrophil-time profile of a typical individual as well as the current patient, based on the parameter values from the Bayesian step.
The following input window asks the user to input a desired target nadir value, and another macro utilizes the individual parameters to calculate a dose predicted to achieve this target. This suggested dose is returned to the user together with a graphical presentation of the expected time-courses following this new dose, as well as following the original first course dose.

\section{Samples of typical program runs}

Fig. 3 shows the estimation dialogue of the tool, where the dose and neutrophils sample information has been input, and the Bayesian process has returned the individual parameters. The graph shows the predicted neutrophil-time profile of a typical individual as well as the patient at hand. The actual measured neutrophils counts are also included in the graph, for the user to judge the fit of the parameters to the data. In Fig. 4 the dose estimation dialogue show and example where a desired nadir target of $1 \times 10^{9}$ cells/L has been chosen, and a dose of $182 \mathrm{mg}$ has been suggested. The graph depicts the predicted neutrophil-time profile of the current dose vs the suggested dose. Fig. 5 shows the worksheet kept in the background containing the parameter matrix, the differential equation system and the Bayesian objective function. Not shown in this figure is the matrix output by the differential equation solver.

The computation performance of the MS Excel tool was evaluated with comparison to the POSTHOC function in NONMEM v6 [29] as the gold standard. Seventy-five patients receiving five consecutive daily $100 \mathrm{mg}$ infusions of etoposide in two courses were simulated in NONMEM. Only data 


\begin{tabular}{|c|c|c|c|c|c|}
\hline \multirow{2}{*}{ PK } & \multicolumn{2}{|l|}{ Population } & \multicolumn{2}{|c|}{ Individual } & \multirow[t]{2}{*}{$\omega$} \\
\hline & tlagp & 0.5 & tlagi & 0.5 & \\
\hline & kap & 0.3 & kai & 0.3 & \\
\hline & $\mathrm{Fp}$ & 0.8 & $\mathrm{Fi}$ & 0.8 & 0.22 \\
\hline & k10p & 0.19 & k10i & 0.19 & \\
\hline & $k 12 p$ & 0.14 & $\mathrm{k} 12 \mathrm{i}$ & 0.14 & 0.21 \\
\hline & k21p & 0.06 & $k 21 i$ & 0.042 & 0.29 \\
\hline & $k 13 p$ & $1 \mathrm{E}-11$ & $\mathrm{k} 13 \mathrm{i}$ & $1 \mathrm{E}-11$ & \\
\hline & k31p & 1 & k31i & 1 & \\
\hline & Vcp & 6 & Vci & 6 & 0.25 \\
\hline & Vssp & 20 & Vssi & 20 & \\
\hline & V3p & 0 & $V_{3} \mathbf{i}$ & 0 & \\
\hline PD & BASE $p$ & 5.45 & BASEi & 5.284934 & 0.42 \\
\hline & GAMMAp & 0.174 & GAMMAi & 0.174 & 0 \\
\hline & SLOPEp & 0.126 & SLOPEi & 0.102013 & 0.4 \\
\hline & ktrp & 0.02963 & ktri & 0.02963 & \\
\hline & MTTp & 135 & MTTi & 135 & 0.14 \\
\hline DOSE & kinf1 & 100 & kinf1 & 100 & \\
\hline & tinf1 & 1 & tinf1 & 1 & \\
\hline & kinf2 & 100 & kinf2 & 100 & \\
\hline & tinf2 & 1 & tinf2 & 1 & \\
\hline & kinf3 & 100 & kinf3 & 100 & \\
\hline & tinf3 & 1 & tinf3 & 1 & \\
\hline & kinf4 & 100 & kinf4 & 100 & \\
\hline & tinf4 & 1 & tinf4 & 1 & \\
\hline & kinf5 & 100 & kinf5 & 100 & \\
\hline & tinf5 & 1 & tinf5 & 1 & \\
\hline
\end{tabular}

\section{Res.variability}

0.15

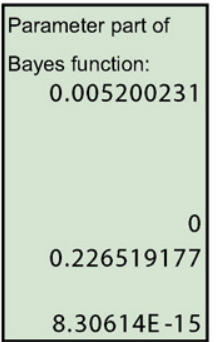

\section{Bayes objective function}

sum(res)

0.58300

\begin{tabular}{|c|c|c|c|}
\hline \multirow{8}{*}{ population } & A1p & 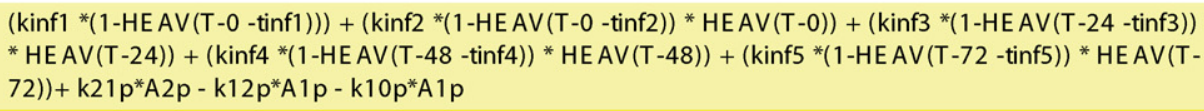 & 0 \\
\hline & $A 2 p$ & $k 12 p^{*} A 1 p-k 21 p^{*} A 2 p$ & 0 \\
\hline & A3p & $k 13 p^{*} A 1 p-k 31 p^{*} A 3 p$ & 0 \\
\hline & A4p & $k_{\operatorname{trp}}^{*} A 4 p^{*}\left(1-\left(S L O P E p^{*} A 1 p / N C p\right)\right)^{*}((B A S E p / A 8 p) \wedge G A M M A p)-k \operatorname{trp}{ }^{*} A 4 p$ & 18.21368 \\
\hline & A5p & $k^{t r p}{ }^{*} A 4 p-k \operatorname{trp} p^{*} A 5 p$ & 18.21368 \\
\hline & A6p & $k \operatorname{trp}{ }^{*} A 5 p-k \operatorname{trp}{ }^{*} A 6 p$ & 18.21368 \\
\hline & A7p & $k \operatorname{trp}{ }^{*} A 6 p-k \operatorname{trp}{ }^{*} A 7 p$ & 18.21368 \\
\hline & A8p & ktrp*A7p - 0.099*A8p & 5.45 \\
\hline \multirow[t]{8}{*}{ Individual } & A1i & 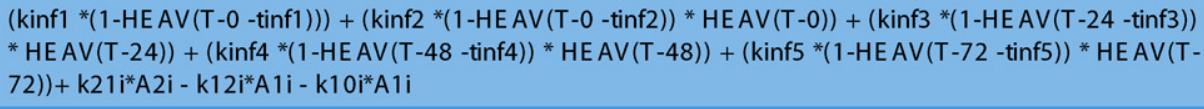 & 0 \\
\hline & $A 2 i$ & $k 12 i^{*} A 1 i-k 21 i^{*} A 2 i$ & 0 \\
\hline & A3i & $\mathrm{k} 13 \mathrm{i}^{*} \mathrm{~A} 1 \mathrm{i}-\mathrm{k} 31 \mathrm{i}^{*} \mathrm{~A} 3 \mathrm{i}$ & 0 \\
\hline & A4i & $k^{2} i^{*} A 4 i^{*}\left(1-\left(S L O P E i^{*} A 1 i N C i\right)\right)^{*}((B A S E i / A 8 i) \wedge G A M M A i)-k t r i * A 4 i$ & 17.66204 \\
\hline & A5i & ktri*A4i - ktri*A5i & 17.66204 \\
\hline & A6i & ktri*A5i - ktri*A6i & 17.66204 \\
\hline & A7i & ktri*A6i - ktri*A7i & 17.66204 \\
\hline & A8i & ktri*A7i $-0.099 * A 8 i$ & 5.284934 \\
\hline
\end{tabular}

Fig. 5 - This figure show the worksheet containing the differential equation system that is solved into a matrix, and the parameters used in the system. The Bayes sum is minimized using the Solver function, by altering the individual parameter estimates thus updating the differential equation system.

from the first course was used as input to the programs. The ability to compute empirical Bayes estimates of individual parameters was compared, as well as the ability to predict future neutrophil measurements in the second course (Table 2). Accuracy and precision were computed by calculat- ing mean prediction error (MPE), mean absolute error (MAE) and root mean squared error (RMSE) [36].

The predictions of individual parameter values as well as sequential course neutrophil counts by the dosing tool and compared to predictions obtained from the POSTHOC function 
Table 2 - Summary statistics of prediction errors of measurements and parameters by NONMEM vs Excel macro.

\begin{tabular}{|c|c|c|c|c|c|c|c|c|}
\hline & \multicolumn{2}{|c|}{ Measurements } & \multicolumn{2}{|c|}{ MTT } & \multicolumn{2}{|c|}{ BASE } & \multicolumn{2}{|c|}{ SLOPE } \\
\hline & NONMEM & Excel & NONMEM & Excel & NONMEM & Excel & NONMEM & Excel \\
\hline Mean error & 0.073 & 0.11 & 0.067 & 0.55 & -0.0013 & 0.11 & 0.0001 & 0.006 \\
\hline Median error & 0.16 & 0.16 & 0.82 & 0.22 & -0.059 & 0.058 & 0.002 & 0.003 \\
\hline S.D. & 1.8 & 1.8 & 8.4 & 7.4 & 0.80 & 0.87 & 0.0018 & 0.045 \\
\hline 1st quartile & -0.72 & -0.63 & -3.7 & -2.9 & -0.51 & -0.45 & -0.022 & -0.020 \\
\hline 3rd quartile & 0.81 & 0.79 & 4.81 & 4.36 & 0.46 & 0.68 & 0.024 & 0.028 \\
\hline MPE & $2.4 \%$ & $3.8 \%$ & $0.20 \%$ & $1.7 \%$ & $0.052 \%$ & $0.44 \%$ & $0.01 \%$ & $2.7 \%$ \\
\hline RMSE & $17 \%$ & $16 \%$ & $12 \%$ & $13 \%$ & $12 \%$ & $13 \%$ & $19 \%$ & $21 \%$ \\
\hline
\end{tabular}

in NONMEM. None of the predicted values differed significantly in accuracy or in precision. Prediction errors were tested for normality by Kolmogorov-Smirnov test. KS was found to range from 0.05 to 0.11 for all parameters and observation errors. Predictions of measurements had a MPE of $2.4 \%$ vs $3.8 \%$ and a RMSE of $17 \%$ vs $16 \%$ for NONMEM and Excel, respectively. The estimation of pharmacodynamic system parameters were also similar; MTT MPE $0.20 \%$ vs $1.7 \%$, RMSE $12 \%$ vs $13 \%$; BASE MPE $0.052 \%$ vs $0.44 \%$ and RMSE $12 \%$ vs $13 \%$. The drug efficacy parameter SLOPE had a MPE of $0.01 \%$ vs $2.7 \%$ and a RMSE of $19 \%$ vs $21 \%$. A summary of the results can be found in Table 2 and Fig. 6 . The small differences in predictions can most likely be attributed to differences in the differential equation solvers, Runge-Kutta method 4,5 vs 5,6 in PopTools and NONMEM, respectively [37]. The mean difference in suggested dose from parameters estimated by Excel compared to NONMEM was $0.2 \%$ with a standard deviation of $8 \%$.

\section{Hardware and software specifications}

Performance has been tested on different PC workstations with MS Excel 2003 installed under Windows XP or 2000. Run times for the individual parameter estimation are approximately 1-3 min, and an additional 1-2 min for the dose recalculation depending on the system performance.

\section{Mode of availability of the program}

The tool is available as an Excel workbook upon request from the authors via electronic mail, and the Excel add-on package Poptools can be obtained from http://www.cse.csiro. $\mathrm{au} /$ poptools.
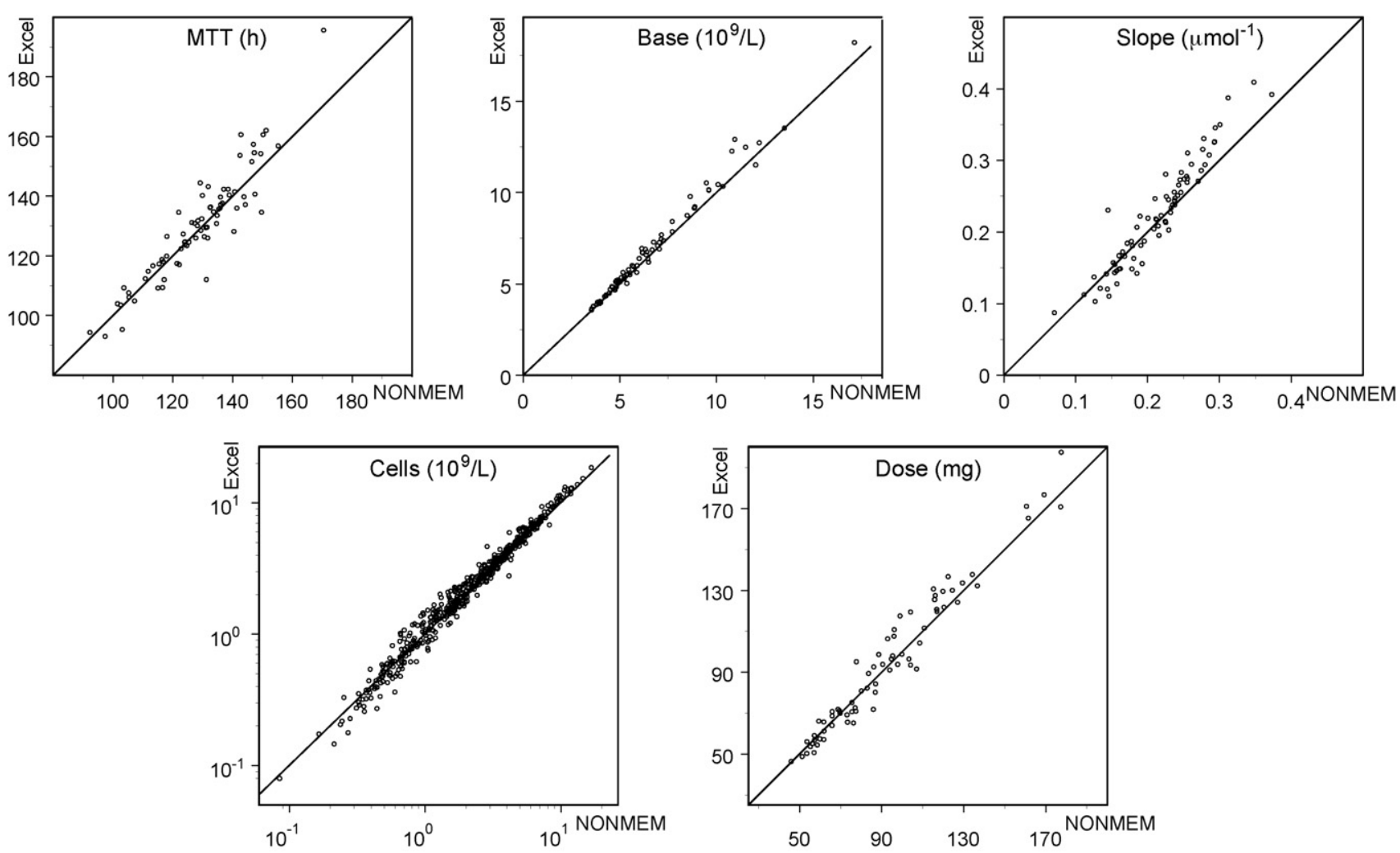

Fig. 6 - Predictions of neutrophil counts, pharmacodynamic parameters and suggested dose of Excel vs NONMEM. 


\section{Discussion}

The model guided dose optimization method in Excel meets the stated prerequisites. It applies a Bayesian predictive method without the need of highly specialized statistical software. It can solve the differential equation system, the parameter estimation produces satisfactory results and the optimization procedure functions well. It has successfully been installed on a number of workstations, but the application in a clinical setting still remains to be tested. The tool can be modified for application on other drugs described by the myelosuppression model, and also for dose adaptation of oral therapy. The main structure rather easily also allows modifications to other models in various therapeutic areas as the Bayesian parameter estimation step is general. Another advantage is that it can handle different types of measures, in this case it was a pharmacokinetic and a pharmacodynamic measure, but therapeutic drug monitoring of parent drug and a metabolite, or possibly two different effect measures on the same chain of response could be implemented. One drawback of the current implementation in Excel is that covariance between individual parameter estimates cannot be accounted for, limiting the use to models without correlations between random effects.

At this time a simulation study is carried out to answer what levels and ratios of inter-individual and inter-occasion variability that renders useful predictions, and when dose adaptation is a good idea. This work should also answer the question whether pharmacokinetics sampling adds any value to predictions if neutrophil measures are made during the first course. These findings should be used as guidelines for deeming the need and utility of model based dose adaptation of anti-cancer drugs, based on modeling results. Published literature models and future findings could then be utilized in clinic care if meeting the criteria. Addition of data from multiple previous courses would need a more complex workbook, but future work will have to answer the predictive importance of accumulated data.

\section{Conflicts of interest}

The authors report no conflicts of interest.

\section{Acknowledgments}

The authors would like to acknowledge the Swedish Cancer Society for financial support. Lena Friberg was supported by Knut and Alice Wallenberg foundation, Sweden and Johan Wallin by the Swedish Pharmaceutical Association.

\section{Appendix A. Supplementary data}

Supplementary data associated with this article can be found, in the online version, at doi:10.1016/j.cmpb.2008.10.011.

\section{REF E RENCES}

[1] G.H. Lyman, D.C. Dale, J. Crawford, Incidence and predictors of low dose-intensity in adjuvant breast cancer chemotherapy: a nationwide study of community practices, J Clin Oncol 21 (24) (2003) 4524-4531.

[2] G.P. Bodey, M. Buckley, Y.S. Sathe, E.J. Freireich, Quantitative relationships between circulating leukocytes and infection in patients with acute leukemia, Ann Intern Med 64 (2) (1966) 328-340.

[3] J. Crawford, D.C. Dale, G.H. Lyman, Chemotherapy-induced neutropenia: risks, consequences, and new directions for its management, Cancer 100 (2) (2004) 228-237.

[4] VePesid ${ }^{\circledR}$ Prescribing Information, Bristol Laboratories, Syracuse, NY, November 1983.

[5] D.A. Cameron, C. Massie, G. Kerr, R.C. Leonard, Moderate neutropenia with adjuvant CMF confers improved survival in early breast cancer, Br J Cancer 89 (10) (2003) 1837-1842.

[6] M. Di Maio, C. Gridelli, C. Gallo, F. Shepherd, F.V. Piantedosi, S. Cigolari, L. Manzione, A. Illiano, S. Barbera, S.F. Robbiati, L. Frontini, E. Piazza, G.P. Ianniello, E. Veltri, F. Castiglione, F. Rosetti, V. Gebbia, L. Seymour, P. Chiodini, F. Perrone, Chemotherapy-induced neutropenia and treatment efficacy in advanced non-small-cell lung cancer: a pooled analysis of three randomised trials, Lancet Oncol 6 (9) (2005) 669-677.

[7] P. Poikonen, T. Saarto, J. Lundin, H. Joensuu, C. Blomqvist, Leucocyte nadir as a marker for chemotherapy efficacy in node-positive breast cancer treated with adjuvant CMF, Br J Cancer 80 (11) (1999) 1763-1766.

[8] S.P. Joel, R. Shah, P.I. Clark, M.L. Slevin, Predicting etoposide toxicity: relationship to organ function and protein binding, J Clin Oncol 14 (1) (1996) 257-267.

[9] L. Nguyen, E. Chatelut, C. Chevreau, B. Tranchand, I. Lochon, J.M. Bachaud, A. Pujol, G. Houin, R. Bugat, P. Canal, Population pharmacokinetics of total and unbound etoposide, Cancer Chemother Pharmacol 41 (2) (1998) 125-132.

[10] S.G. Arbuck, H.O. Douglass, W.R. Crom, P. Goodwin, Y. Silk, C. Cooper, W.E. Evans, Etoposide pharmacokinetics in patients with normal and abnormal organ function, J Clin Oncol 4 (11) (1986) 1690-1695.

[11] C.F. Stewart, S.G. Arbuck, R.A. Fleming, W.E. Evans, Changes in the clearance of total and unbound etoposide in patients with liver dysfunction, J Clin Oncol 8 (11) (1990) 1874-1879.

[12] C.F. Stewart, R.A. Fleming, S.G. Arbuck, W.E. Evans, Prospective evaluation of a model for predicting etoposide plasma protein binding in cancer patients, Cancer Res 50 (21) (1990) 6854-6856.

[13] M.E. de Jonge, A.D. Huitema, J.H. Schellens, S. Rodenhuis, J.H. Beijnen, Individualised cancer chemotherapy: strategies and performance of prospective studies on therapeutic drug monitoring with dose adaptation: a review, Clin Pharmacokinet 44 (2) (2005) 147-173.

[14] E. Evene, E. Chatelut, B. Tranchand, P. Canal, I. Lochon, A. Iliadis, C.J. Ardiet, Bayesian estimation of pharmacokinetic parameters of etoposide, Bull Cancer 84 (7) (1997) 699-703.

[15] B. Tranchand, C. Amsellem, E. Chatelut, G. Freyer, A. Iliadis, B. Ligneau, V. Trillet-Lenoir, P. Canal, I. Lochon, C.J. Ardiet, A limited-sampling strategy for estimation of etoposide pharmacokinetics in cancer patients, Cancer Chemother Pharmacol 43 (4) (1999) 316-322.

[16] A. Rousseau, P. Marquet, J. Debord, C. Sabot, G. Lachatre, Adaptive control methods for the dose individualisation of anticancer agents, Clin Pharmacokinet 38 (4) (2000) 315-353.

[17] G. Levy, Predicting effective drug concentrations for individual patients. Determinants of pharmacodynamic variability, Clin Pharmacokinet 34 (4) (1998) 323-333. 
[18] F. Leger, W.J. Loos, R. Bugat, R.H. Mathijssen, M. Goffinet, J. Verweij, A. Sparreboom, E. Chatelut, Mechanism-based models for topotecan-induced neutropenia, Clin Pharmacol Ther 76 (6) (2004) 567-578.

[19] M. Sandstrom, H. Lindman, P. Nygren, M. Johansson, J. Bergh, M.O. Karlsson, Population analysis of the pharmacokinetics and the haematological toxicity of the fluorouracil-epirubicin-cyclophosphamide regimen in breast cancer patients, Cancer Chemother Pharmacol 58 (2) (2006) 143-156.

[20] L.E. Friberg, M.O. Karlsson, Mechanistic models for myelosuppression, Invest New Drugs 21 (2) (2003) 183-194.

[21] L.E. Friberg, A. Henningsson, H. Maas, L. Nguyen, M.O. Karlsson, Model of chemotherapy-induced myelosuppression with parameter consistency across drugs, J Clin Oncol 20 (24) (2002) 4713-4721.

[22] M.O. Karlsson, T. Anehall, L.E. Friberg, A. Henningsson, C. Kloft, M. Sandstrom, R. Xie, Pharmacokinetic/pharmacodynamic modelling in oncological drug development, Basic Clin Pharmacol Toxicol 96 (3) (2005) 206-211.

[23] C. Kloft, J. Wallin, A. Henningsson, E. Chatelut, M.O. Karlsson, Population pharmacokinetic-pharmacodynamic model for neutropenia with patient subgroup identification: comparison across anticancer drugs, Clin Cancer Res 12 (18) (2006) 5481-5490.

[24] J.E. Latz, M.O. Karlsson, J.J. Rusthoven, A. Ghosh, R.D. Johnson, A semimechanistic-physiologic population pharmacokinetic/pharmacodynamic model for neutropenia following pemetrexed therapy, Cancer Chemother Pharmacol 57 (4) (2006) 412-426.

[25] J.E. Latz, J.J. Rusthoven, M.O. Karlsson, A. Ghosh, R.D. Johnson, Clinical application of a semimechanistic-physiologic population PK/PD model for neutropenia following pemetrexed therapy, Cancer Chemother Pharmacol 57 (4) (2006) 427-435.

[26] M. Sandstrom, H. Lindman, P. Nygren, E. Lidbrink, J. Bergh, M.O. Karlsson, Model describing the relationship between pharmacokinetics and hematologic toxicity of the epirubicin-docetaxel regimen in breast cancer patients, $\mathrm{J}$ Clin Oncol 23 (3) (2005) 413-421.
[27] C. van Kesteren, A.S. Zandvliet, M.O. Karlsson, R.A. Mathot, C.J. Punt, J.P. Armand, E. Raymond, A.D. Huitema, C. Dittrich, H. Dumez, H.H. Roche, J.P. Droz, M. Ravic, S.M. Yule, J. Wanders, J.H. Beijnen, P. Fumoleau, J.H. Schellens, Semi-physiological model describing the hematological toxicity of the anti-cancer agent indisulam, Invest New Drugs 23 (3) (2005) 225-234.

[28] E.K. Hansson, J.E. Wallin, M. Sandström, M.O. Karlsson, L.E. Friberg, Comparison of inter-occasion and inter-individual variability in chemotherapy-induced myelosuppression, PAGE, in: Abstracts of the Annual Meeting of the Population Approach Group in Europe, 2008 (Abstract 1328).

[29] S.L. Beal, L.B. Sheiner, NONMEM Users Guides, GloboMax, Inc., Hanover, MD, 1989-2006.

[30] Greg Hood, PopTools, CSIRO, 2007, Internet resource: www.cse.csiro.au/poptools.

[31] G. Toffoli, G. Corona, R. Sorio, I. Robieux, B. Basso, A.M. Colussi, M. Boiocchi, Population pharmacokinetics and pharmacodynamics of oral etoposide, Br J Clin Pharmacol 52 (5) (2001) 511-519.

[32] G.E. Cartwright, J.W. Athens, M.M. Wintrobe, The kinetics of granulopoiesis in normal man, Blood 24 (1964) 780-803.

[33] T. Bayes, An essay towards solving a problem in the doctrine of chances, Philos Trans R Soc 53 (1763) 420-426.

[34] R.W. Jelliffe, A. Schumitzky, M. Van Guilder, M. Liu, L. Hu, P. Maire, P. Gomis, X. Barbaut, B. Tahani, Individualizing drug dosage regimens: roles of population pharmacokinetic and dynamic models, Bayesian fitting, and adaptive control, Ther Drug Monit 15 (5) (1993) 380-393.

[35] L.B. Sheiner, S. Beal, B. Rosenberg, V.V. Marathe, Forecasting individual pharmacokinetics, Clin Pharmacol Ther 26 (3) (1979) 294-305.

[36] L.B. Sheiner, S.L. Beal, Some suggestions for measuring predictive performance, J Pharmacokinet Biopharm 9 (4) (1981) 503-512.

[37] J.R. Dormand, P.J. Prince, A family of embedded Runge-Kutta formulae, J Comput Appl Math 6 (1980) 19-26. 